\title{
Self care system for Heart Failure out of hospital patients
}

\author{
E Villalba, I Peinado, MT Arredondo \\ Life Supporting Technologies \\ Technical University of Madrid \\ Madrid, Spain \\ \{evmora, ipeinado,mta\}@1st.tfo.upm.es
}

\author{
MT Meneu, P Sala, S Guillen \\ Health and Wellbeing Technologies R\&D \\ ITACA Institute \\ Valencia, Spain \\ sguillen@itaca.upv.es
}

\begin{abstract}
CUORE is a Heart Failure (HF) Disease Assessment System that makes use of innovative approaches, based on Information Technologies (IT) and portable monitoring devices, for the continuous assessment of HF progression and cardiovascular risk stratification. The system valuates the cardiac condition integrating patient data from different sources with special emphasis in the information obtained for ECG processing. Rather than just evaluate the cardiovascular status, the system also aims to motivate patients to have an active role in their health management and to improve their cardiac condition by promotion of an active lifestyle. In order to make the system usable the methodology adopted to create the final solution is iterative and it involves users in all stages. This paper presents the conceptualization of CUORE as a solution to self care for heart failure out of hospital patients.
\end{abstract}

Keywords- user interaction, health monitoring, personalized applications, heart failure.

\section{INTRODUCTION}

Cardiovascular diseases (CVD) are the leading cause of death in Western World. They provoke $40 \%$ of all deceases [1]. Heart Failure, which is considered the paradigm of chronic CVD, mainly affects people over 65 years. Besides, the aging population increases yearly [2]. Within this context, solutions such as COURE play a role of major importance.

CUORE will provide Heart Failure patients with a usable and fluent channel of communication with health professionals in order to support them to manage comorbidities related to their condition. The proposed solution monitors daily vital info such as BP, weight and HR. Furthermore, it aims to motivate patients and to improve adherence to the technology and medical protocols. The system will be used at patients' home within their daily lives. The professionals will interact through a ubiquitous connection based on a secure web portal.

\section{METHODS}

The final solution will interact with older people with chronic disease. Thus, the design is crucial. The methodology

This work has been supported in part by the ITEA Program within UADU project (ITEA 05003)

$\mathrm{N}$ focuses on observing and interviewing real users in all stages of the global process [3].
Therefore, there are intermediate validations with real users before the final implementation. Thus, as figure 1 illustrates, there are 4 phases.

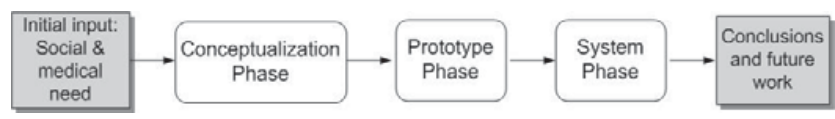

Figure 1. Process applied to design, develop and validate the solution

The initial input is the social and medical need of solutions to assist people living with a chronic disease, especially when they have special needs of interaction (i.e. elderly). The conceptualization phase plays an importance role to assure the success of these solutions. This paper focuses on the Conceptualization Phase. This initial phase aims to create a preliminary non-functional prototype to validate the concept with involved stakeholders, as shown in figure 2. Afterwards, a prototype is developed and validate also with stakeholders.

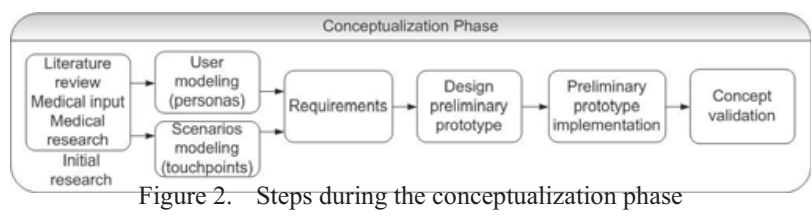

Initially, similar solutions where studied and literature about interaction, heart failure and living with a chronic disease was revised to understand the context and needs [4]. The modeling focused on users, named personas; and scenarios [5]. Afterwards, a list of requirements culminated with a prototype design which is implemented in a nonfunctional mock-up. This concept is then evaluated with three stakeholders: cardiologists, nurses and heart failure patients. Next sections explain in details this process.

\section{MOdELS: PERSONAS \& SCENARIOS}

\section{A. Personas}

COURE has three main actors that interact with the system. For each actor a persona (i.e. archetype that contains the main characteristics of the target group) is created.

After revising all literature about heart failure population and checking previous studies in which validation with real

PERVASIVEHEALTH 2008, 30 Jan - 1 Feb. Tampere, Finland

Copyright (C) 2008 ICST 978-963-9799-15-8

DOI 10.4108/ICST.PERVASIVEHEALTH2008.4057 
users where carried out [4], the final archetype user is a 72 years old man named Carlos Gómez. He is retired and claims for a sense of independency. He is conscious of his heart status. His cardiologist is Dr. Casas, 52 years old, who aims to feel reassurance for his patients and the possibility of early diagnostic of a decompensation.

Marta Besteiro is the nurse in charge of the assessment out of hospital. She is worry about the trend of all her patients and she asks for tools to give them a better support and understanding of their own situation and health status.

\section{B. Scenarios}

Scenarios recreate a day in the life for each persona [5].

Carlos wakes up at 8:00 a.m. every morning. After going to the bathroom, he checks his PDA to see if he has new messages. He has two new messages. The first one warns him that his medication has changed. The second one is a reminder to fill in the monthly questionnaire to value his feel of quality of life. Thus, he answers 5 short questions and check which drug has changed. Afterwards, Carlos enters the daily activities guide that is personalized to his routine. Today, he has to measure his weight, blood pressure and ECG. For that, he has at his home all necessary devices that have been designed for him. Thanks to this solution, he feels that he is taking care of his health. Besides, his PDA offers an educational tool with important information about heart failure and healthy lifestyle, nutrition and routines such as light physical activities.

Marta and Dr. Casas check the portal in the evening. They had a lot of patients today. Looking at the answers of the monthly questionnaire, they notice that Mr. Gómez seems worrier than previous months. Therefore, they write a message to ask him to come to the hospital the coming week.

\section{REQUIREMENTS, FUNCTIONAL AND INTERACTION DESIGN}

The main user requirements are listed in the following bullets:

- $\quad$ Very simple and intuitive to start

- $\quad$ Easy to use

- $\quad$ Very fast to perform daily activities and always ready when needed

- Adaptable to personal routines

- $\quad$ Need of guide for use, for measurements and help functionalities

- $\quad$ Error prevention and recovery

The functionalities for the final user are listed below:

- Medical data gathering from all sensors around the user

- Treatment consultation for drugs dosage and frequency/periodicity
- User guide for Weight, Blood Pressure and ECG/HR measurement, including help functionalities

- Questionnaires to report health status to the professionals

- Medical agenda with reminders, next visit to the hospitals, etc.

- Message box for managing all messages received from professionals (e.g. read/unread or priorities)

- Well-being practise and understanding your heart and its care functionality

The professional will access all data through a web portal with login and password. Main functionalities are:

- All patients overview with main status and evolution, highlighting worsening

- $\quad$ Patient edition

- Patient record visualization

- Medical data consultation

- Treatment consultation and edition (only cardiologist)

- Professionals community - information interchange among professionals, with annotations, "post-it", etc.

- Messages to patients with filtering

- Tools for visualising vital signals (e.g. ECG) or trends

Besides, the system offers the following common functionalities:

- Data synchronization among home stations and servers

- Data managing for extracting information about the status of all patients. Not only in terms of health status, but also regarding their behaviour, and technical status on the sensors and devices

The interaction design culminates with workflows for all functionalities [5][6][7]. For instance, figure 3 shows the workflow for the measurement and questionnaire guide.

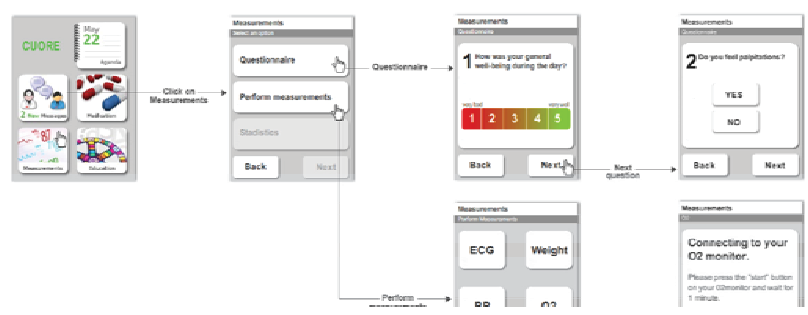


Figure 4 illustrates the workflow for medications.

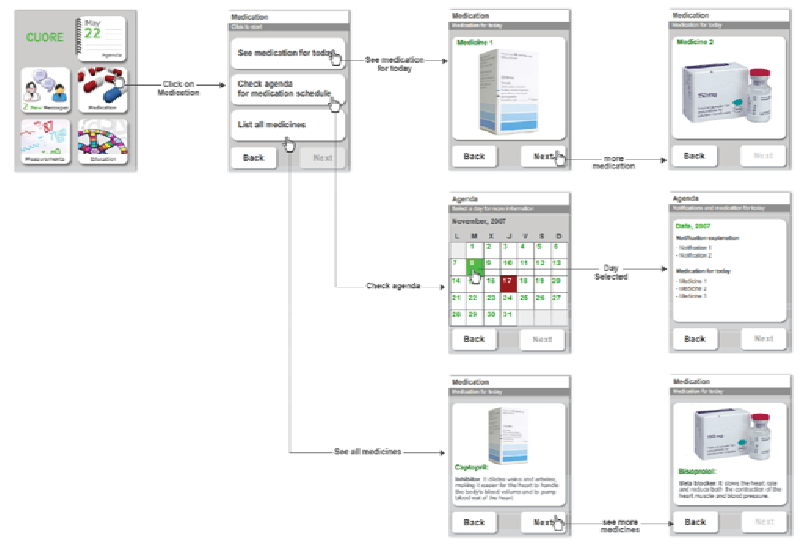

Figure 4. Interaction workflow for measurements and questionnaire

Thusly, next section explains the conceptual prototype implemented. The PDA mock-up is first implemented in FLASH [8] and the Portal in HTML and CSS [9].

\section{Conceptual Prototype}

After the preliminary design, a preliminary prototype has been implemented. The proposed system is divided into three main areas: the user interaction system, the common platform which contains all services; and the professional interaction.

The user interaction system comprises all electronics and the user interaction device. The sensors and electronics to monitor patients in their daily routines:

- Blood pressure cuff

- Weight scale

- ECG/HR monitor

- Accelerometer

The user interaction device is implemented in a PDA which guides, educates, motivates and allows all necessary feedback.

The common platform assures the connectivity and security. This platform enables communication and data synchronization among all modules in the global distributed system.

Professionals interact in a ubiquitous way through a secure web portal to follow all patients' trends and to manage their protocols and treatments. Besides, it permits the interchange of information and knowledge among professionals.

Figure 2 illustrates the holistic adopted solution.

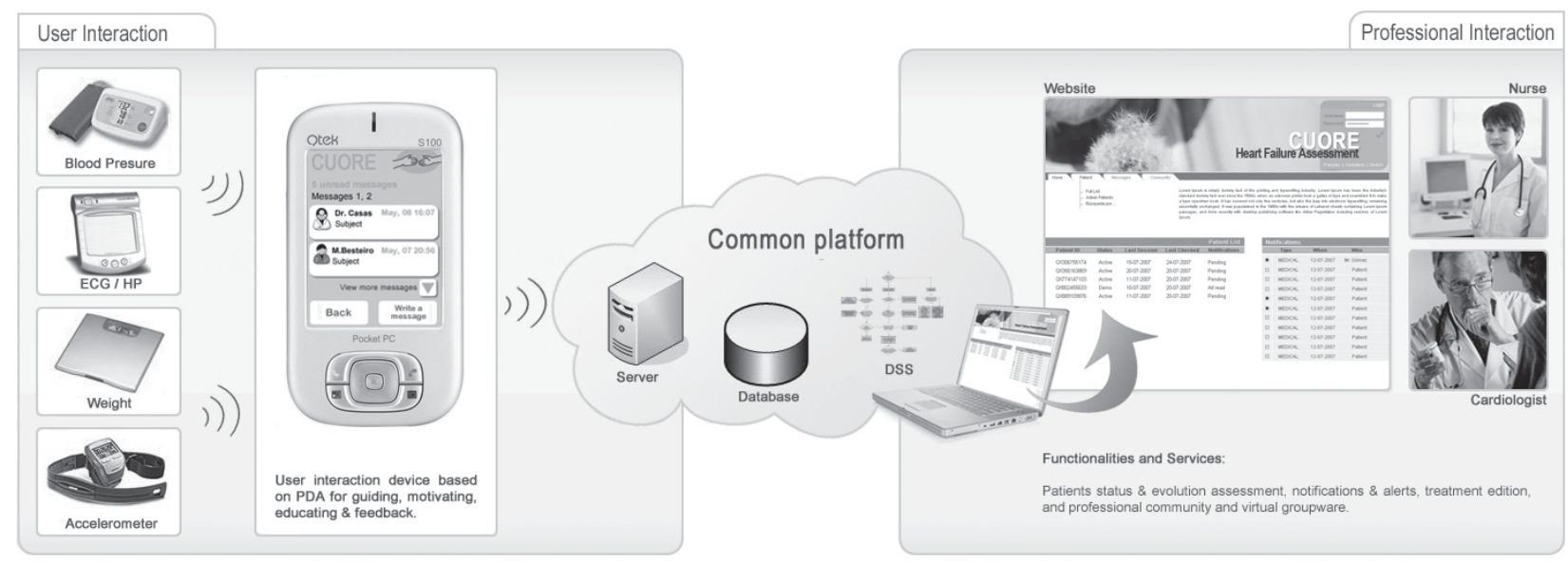

Figure 5. Scheme for the solution to assess heart failure patients out of hospital

The daily routine data are processed and evaluated for the detection of functional capacity, heart failure worsening and other complications. Motivation strategies must be taken into account in order to provide patients with relevant information, according to their physical and psychological status.

The PDA application is divided into 5 functional areas: Agenda, Messages, Medication, Measurements and Education.
Agenda reminds the user all measurements which are to be performed to monitor the patient's health status. Messages area stores important information which has been sent by professionals. Medication are has two aims, first reminds of medication for a concrete day, with dosage; and second information of drugs (e.g. main effect).

In this prototype, the possible measurements are ECG, weight, blood pressure and oxygen saturation. Education offers relevant information of living with chronic diseases, 
good practices for nutrition and active life. With this Education area the system will aware the user of all benefits derived from adherence to protocols. Besides it will motivate him to keep on a healthy lifestyle.

The professional portal has been implemented in HTML and CSS following WEB 2.0 principles [9]. Following figure shows two examples.

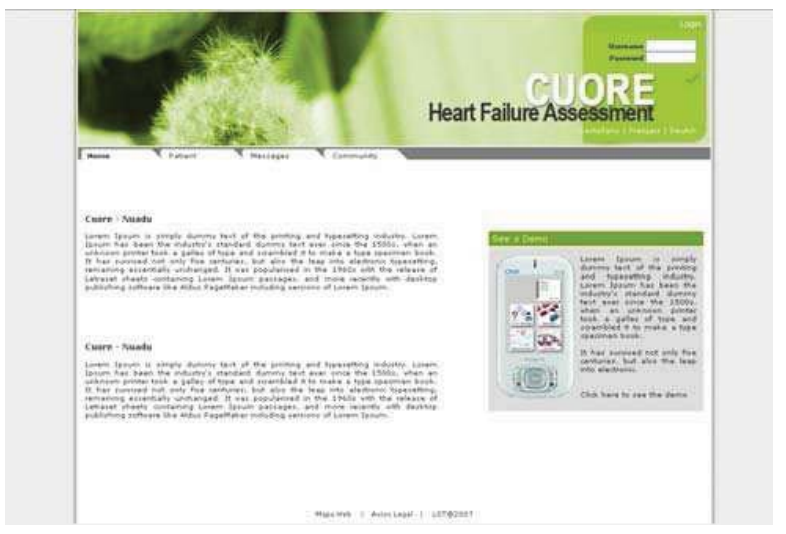

Figure 6. Home web page for professionals' portal

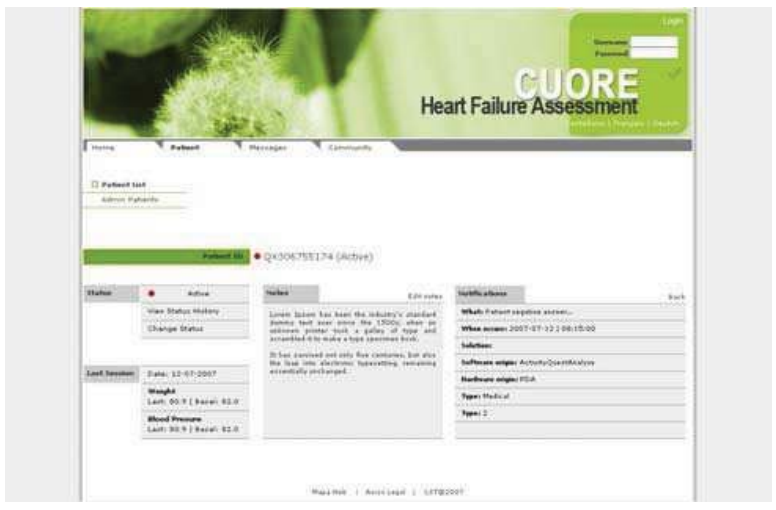

Figure 7. Patient's page with all data

\section{CONCLUSIONS AND FUTURE WORK}

These preliminary prototypes have been validated by experts, more concretely by two cardiologists and three user interaction and usability experts. The testing performed by experts has followed the ten Nielsen's Heuristics [10]. The patient station was considered easy to use. Nevertheless, all experts agreed on the need to use bigger screens, i.e. TVs. However, these devices do not allow patients' mobility, which is one of the user's experience goals. The professional portal was considered of high usability.

Future work comprises the functional prototype implementation. First of all, models, functionalities and design are to be revised accordingly to stakeholders' insights. A functional validation is to be implemented and validated in a pilot at the hospital with 10 to 20 patients using the system at their homes for 2 weeks. Final revised system will be then refined.
The preliminary results show promising in terms of the interaction modality implemented. However, a detailed analysis in order to enhance individuals experience and incorporate this system into their routine is still lacking. This entails an in depth study of diverse behavior components towards e-health in order to create a tailored communication framework, which boosts motivation of patients to use such systems and truly incorporate them to their daily activities. A framework to be followed considers the analysis of different variables [11].

To conclude, in the new paradigm of Ambient Intelligence, we strongly believe that in the future this kind of systems will represent an important part of the daily activity of this sort of patients, supporting a better quality of life and helping to prevent and to treat chronic diseases.

\section{ACKNOWLEDGMENT}

This work has succeeded thanks to the close collaboration with Hospital San Carlos of Madrid, Spain and ITACA Institute, (Valencia, Spain). COURE is an integrated system of the NUADU project (ITEA 05003).

\section{REFERENCES}

[1] World Health Organization. "The Atlas of Heart Disease and Stroke". Edited by J. Mackay and G. Mensah, 2004.

[2] Eurostat (2005) Population projections 2004-2050.

[3] ISTAG Report on Experience \& Application Research. "Involving users in the Development of Ambient Intelligent. Edited by European Communities, 2004. ISBN: 92-894-8136-3

[4] Villalba E, Peinado I, Arredondo MT. "User Interaction Design for a Wearable and IT based Heart Failure System". HCI Internationa 2007. ISBN: 978-3-540-73738-4.

[5] Cooper A, "About Face 3.0 The Essentials of Interaction Design". Edited by Wiley Publishing, Inc. 2007. ISBN: 978-0-470-08411-3.

[6] Moggridge B, Designing Interactions. The MIT Press, Cambridge, Massachusetts 2007. ISBN: 978-0-262-13474-3.

[7] C. Zwick, B. Schmitz, K. Kuehl, "Designing for Small Screens". Edited by AVA publishing SA, 2005. ISBN: 2-940373-07-8.

[8] K. Ulrich, Macromedia Flash MX 2004 for Windows and Macintosh. Edited by Pearson Prentice Hall, 2006. ISBN 8420548464.

[9] P. Haine, HTML Mastery: Semantics, Standards, and Styling. Edited by Friends of ED, 2007.

[10] Dumas JS, Redish JC, "A practical guide to usability testing", Edited by Intellect Books, 1999. ISBN: 1-84150-020-8.

[11] del Hoyo-Barbolla E, Arredondo MT, Ortega M, Fernández N, Villalba-Mora E. "A new approach to model the adoption of ehealth. Proceedings" 13th IEEE Mediterranean Electrotechnical Conference. Benalmádena (Spain) May 2006. ISBN: 1-4244-00880 